\title{
The clinical utility of lung clearance index in early cystic fibrosis lung disease is not impacted by the number of multiple-breath washout trials
}

\author{
Rachel E. Foong ${ }^{1,2,3}$, Alana J. Harper ${ }^{1}$, Billy Skoric $\mathbb{1}^{4,5}$, Louise King ${ }^{4,5}$, \\ Lidija Turkovic $^{1}$, Miriam Davis ${ }^{6}$, Charles C. Clem ${ }^{6}$, Tim Rosenow (1) 1 , \\ Stephanie D. Davis ${ }^{6}$, Sarath Ranganathan ${ }^{5}$, Graham L. Hall ${ }^{1,3}$ and \\ Kathryn A. Ramsey (1) ${ }^{1,7}$ on behalf of AREST CF $^{8}$
}

Affiliations: ${ }^{1}$ Telethon Kids Institute, Subiaco, Australia. ${ }^{2}$ The Hospital for Sick Children, Toronto, ON, Canada. ${ }^{3}$ School of Physiotherapy and Exercise Science, Curtin University, Bentley, Australia. "Murdoch Children's Research Institute, Parkville, Australia. ${ }^{5}$ Dept of Respiratory Medicine, The Royal Children's Hospital, Parkville, Australia. 'Section of Pediatric Pulmonology, Allergy and Sleep Medicine, Dept of Pediatrics, Riley Hospital for Children, Indiana University School of Medicine, Indianapolis, IN, USA. ${ }^{7}$ Paediatric Respiratory Medicine, Inselspital, University of Bern, Bern, Switzerland. ${ }^{8}$ The full membership of the Australian Respiratory Early Surveillance Team for Cystic Fibrosis (AREST CF) is available at www.arestcf.org.

Correspondence: Graham L. Hall, Children's Lung Health, Telethon Kids Institute, 100 Roberts Road, Subiaco, WA 6008, Australia. E-mail: Graham. Hallatelethonkids.org.au

ABSTRACT The lung clearance index (LCI) from the multiple-breath washout (MBW) test is a promising surveillance tool for pre-school children with cystic fibrosis (CF). Current guidelines for MBW testing recommend that three acceptable trials are required. However, success rates to achieve these criteria are low in children aged $<7$ years and feasibility may improve with modified pre-school criteria that accepts tests with two acceptable trials. This study aimed to determine if relationships between LCI and clinical outcomes of CF lung disease differ when only two acceptable MBW trials are assessed.

Healthy children and children with CF aged 3-6 years were recruited for MBW testing. Children with $\mathrm{CF}$ also underwent bronchoalveolar lavage fluid collection and a chest computed tomography scan.

MBW feasibility increased from $46 \%$ to $75 \%$ when tests with two trials were deemed acceptable compared with tests where three acceptable trials were required. Relationships between MBW outcomes and markers of pulmonary inflammation, infection and structural lung disease were not different between tests with three acceptable trials compared with tests with two acceptable trials.

This study indicates that pre-school MBW data from two acceptable trials may provide sufficient information on ventilation distribution if three acceptable trials are not possible.

@ERSpublications

Two multiple-breath washout test trials are sufficient to determine lung clearance index in early CF lung disease http://ow.ly/YHxu30hnrl2

Cite this article as: Foong RE, Harper AJ, Skoric B, et al. The clinical utility of lung clearance index in early cystic fibrosis lung disease is not impacted by the number of multiple-breath washout trials. ERJ Open Res 2018; 4: 00094-2017 [https://doi.org/10.1183/23120541.00094-2017].

Received: July 262017 | Accepted after revision: Nov 242017

Support statement: Funding for the AREST CF program and this project was obtained from the Cystic Fibrosis Foundation Therapeutics (SLY04A0, STICK09A0 and HALL14A0), the National Health and Medical Research Council of Australia (NHMRC) (APP513730 and Centre of Research Excellence 1000896). K.A. Ramsey (APP1088389) and G.L. Hall (APP1025550) are NHMRC Research Fellows. Funding information for this article has been deposited with the Crossref Funder Registry.

Conflict of interest: Disclosures can be found alongside this article at openres.ersjournals.com

Copyright $\odot$ ERS 2018. This article is open access and distributed under the terms of the Creative Commons Attribution Non-Commercial Licence 4.0. 


\section{Introduction}

The lung clearance index (LCI) is a measure of ventilation distribution obtained from the multiple-breath washout (MBW) test [1]. As the MBW test uses relaxed tidal breathing without the requirement of any maximal effort, it is an attractive test for infants and pre-school-aged children [2]. LCI is abnormal in a significant proportion of pre-school children with cystic fibrosis (CF), and correlates with the presence and extent of structural lung disease detected via chest computed tomography (CT) [3, 4] and lower respiratory tract inflammation and infection [5-7]. These data suggest that MBW is sensitive to underlying lung disease in young children with CF.

There is growing interest in the use of MBW in paediatric CF clinical care, research and therapeutic trials. The 2013 European Respiratory Society (ERS)/American Thoracic Society (ATS) consensus statement for inert gas washout testing recommends that at least three technically acceptable trials be performed for the test to be considered acceptable; however, this document also states that data may be reported if only two acceptable trials are available [1]. MBW testing in pre-school children may require many attempts to obtain three technically acceptable trials, thereby reducing feasibility in the clinic setting [8]. Due to the low feasibility associated with obtaining three successful MBW trials in pre-school children, many studies already report data from tests with only two acceptable trials and have noted increased feasibility using this approach $[9,10]$. These studies suggest that MBW test criteria may need to be amended for the pre-school age range to allow data to be reported from tests with only two acceptable MBW trials.

Ideally, it is essential that improved MBW feasibility does not reduce sensitivity and specificity to detect underlying disease. This study aimed to determine if relationships between LCI and clinical outcomes of CF lung disease are different when only two acceptable MBW trials are assessed. We hypothesise that feasibility would improve when two acceptable MBW trials were required compared with three acceptable trials, and that associations between LCI and clinical markers of CF lung disease would not differ between tests with three versus two MBW trials. We examined this by performing a paired analysis between MBW outcomes from the first two trials versus all trials in visits whereby at least three acceptable trials were obtained. Furthermore, we examined group differences in MBW outcomes between visits with at least two acceptable trials versus visits with at least three acceptable trials to determine if changing the trial acceptability criteria impacted associations with clinical markers of disease.

\section{Methods}

Study population

Children with CF enrolled in the Australian Respiratory Early Surveillance Team for Cystic Fibrosis (AREST CF) early surveillance programme in Princess Margaret Hospital (Perth, Australia) and The Royal Children's Hospital (Parkville, Australia) between November 2012 and June 2016 were eligible to participate in this study. This programme starts following diagnosis via newborn screening at 3 months and involves annual age-appropriate lung function testing a few days prior to the child's annual review visit, which includes bronchoalveolar lavage (BAL) fluid collection via bronchoscopy and/or chest CT until the age of 6 years. As part of a larger multicentre longitudinal study, MBW data from children with CF at the Riley Hospital for Children (Indianapolis, IN, USA) recruited between May 2015 and June 2016 were also included in this study. These children underwent MBW testing using the same equipment and protocol as the AREST CF centres, and attended a study visit for MBW testing scheduled around a bronchoscopy and chest CT. Children with no history of respiratory disease, pre-term birth or asthma medication use were recruited to perform MBW testing only from the community in Perth and Melbourne in Australia and in Indianapolis in the USA. The ethics committee of each institution approved the study and parents gave informed consent to each aspect of the study.

\section{Multiple-breath washout test}

MBW was performing using $100 \%$ oxygen to washout resident nitrogen from the lung with the Exhalyzer D system (EcoMedics, Duernten, Switzerland) and associated Spiroware software (version 3.1.6) [3]. Children performed the test seated using a noseclip and low dead space snorkel-like mouthpiece (EcoMedics). At each visit children aimed to achieve three acceptable trials according to ERS/ATS consensus criteria [1] and underwent qualitative quality control assessment [11]. As per the current recommendation, MBW trials with functional residual capacity (FRC) values that differed by $>25 \%$ from the median were excluded [1]. Measures of FRC, LCI, the first moment ratio (M1/M0) and the second moment ratio (M2/M0) were reported. MBW tests were categorised as 1) all acceptable data (two or more acceptable trials): MBW tests with two or more acceptable trials; 2) three or more acceptable trials: MBW tests with three or more acceptable trials; and 3) two acceptable trials: MBW tests with only two acceptable trials. 
Bronchoalveolar lavage

A bronchoscopy was performed within 3 days after MBW testing for the collection of BAL fluid in children with CF only for the detection of bacteria, viruses and fungi using standard microbiological culture techniques, and for the quantification of total and differential inflammatory cell counts and measurement of free neutrophil elastase activity [12]. A colony count for a specific organism (excluding mixed oral flora) $>10^{4} \mathrm{CFU} \cdot \mathrm{mL}^{-1}$ was defined as a pulmonary infection. Bacterial pathogens known to elicit a pro-inflammatory response when isolated from the lower respiratory tract (Pseudomonas aeruginosa, Staphylococcus aureus, Haemophilus influenzae, Streptococcus pneumonia and Aspergillus species) were described as pro-inflammatory pathogens [13].

\section{Chest computed tomography}

Chest CT under general anaesthesia was performed prior to bronchoscopy on children with CF. Volumetric inspiratory scans were obtained at a positive airway opening pressure of $25 \mathrm{cmH}_{2} \mathrm{O}$ and volumetric or limited slice expiratory scans were obtained at an airway opening pressure of $0 \mathrm{cmH}_{2} \mathrm{O}$ as described previously [3]. CT scans were scored using the quantitative Perth-Rotterdam Annotated Grid Morphometric Analysis for Cystic Fibrosis (PRAGMA-CF). The separate proportions of total structural lung disease, bronchiectasis and trapped air were reported [14].

\section{Statistical analysis}

Comparisons of MBW outcomes in children with $\mathrm{CF}$ and healthy children were performed using t-tests, while comparisons of proportional differences were performed using Chi-squared tests. Associations between MBW outcomes with respiratory infection, inflammation and structural lung disease outcomes were calculated using linear mixed effects models with random intercepts for repeated visits to account for clustering. Linear mixed effects models were used to compare the relationships between clinical markers of lung disease with MBW outcomes derived from 1) all trials of children with CF who achieved three or more acceptable trials, 2) the first two trials of children who achieved three or more acceptable trials and 3) the first two trials of children who achieved two or more acceptable trials. Fixed regression coefficients of mixed effects models between two and three MBW trials were compared using the z-test [15]. Sensitivity and specificity were also calculated together with 95\% exact binomial confidence intervals. Analyses were performed using SPSS version 23 (IBM, St Leonards, Australia) and Stata version 13.0 (StataCorp, College Station, TX, USA).

\section{Results}

\section{Study population}

MBW testing was performed in 90 children with CF (149 visits) and 56 healthy children (72 visits) aged 3-6 years (figure 1). There were no differences in age, height or weight z-scores and FRC between CF and healthy children. Ventilation distribution outcomes (LCI, M1/M0 and M2/M0) were significantly higher in children with CF compared with healthy controls $(\mathrm{p}<0.001)$ (table 1).

The overall success rate to obtain at least three acceptable MBW trials in pre-school children was $46 \%$. Overall feasibility to obtain at least two acceptable MBW trials was $75 \%$, which was significantly higher

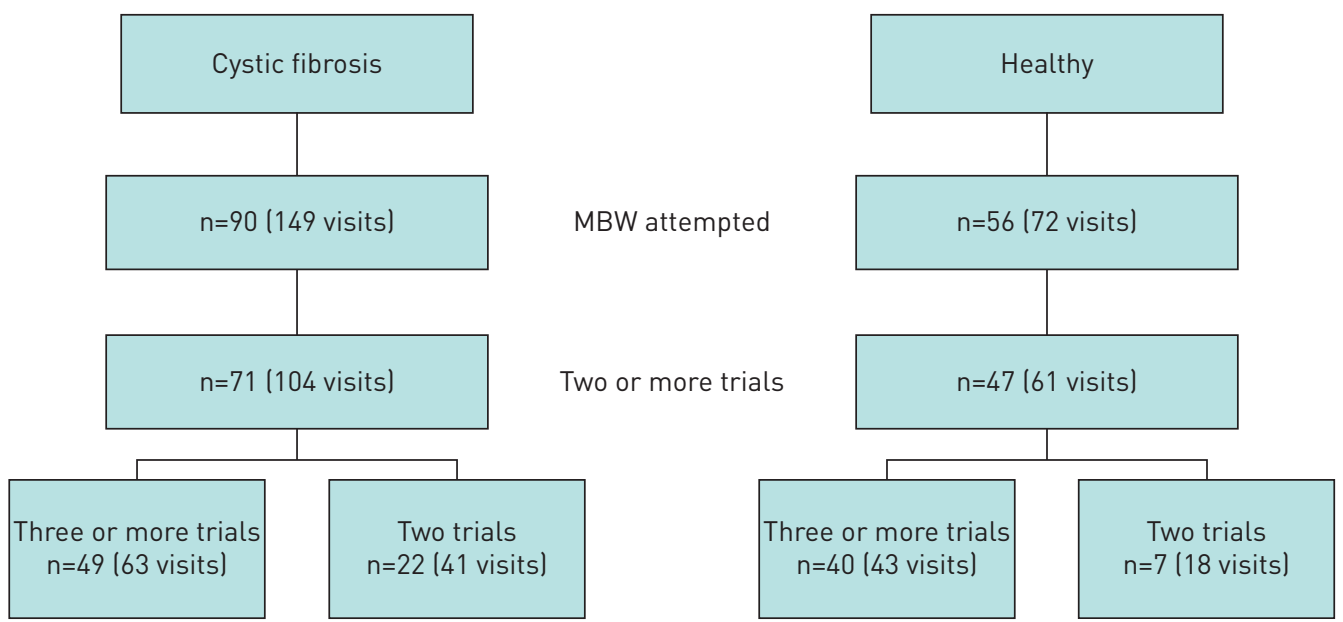

FIGURE 1 Flowchart of children with cystic fibrosis and healthy children enrolled in this study. MBW: multiple-breath washout. 
TABLE 1 Demographics of children with cystic fibrosis (CF) and healthy children for all visits with two or more acceptable multiple-breath washout (MBW) trials, visits with three or more acceptable MBW trials and visits with only two acceptable MBW trials obtained within a single testing session

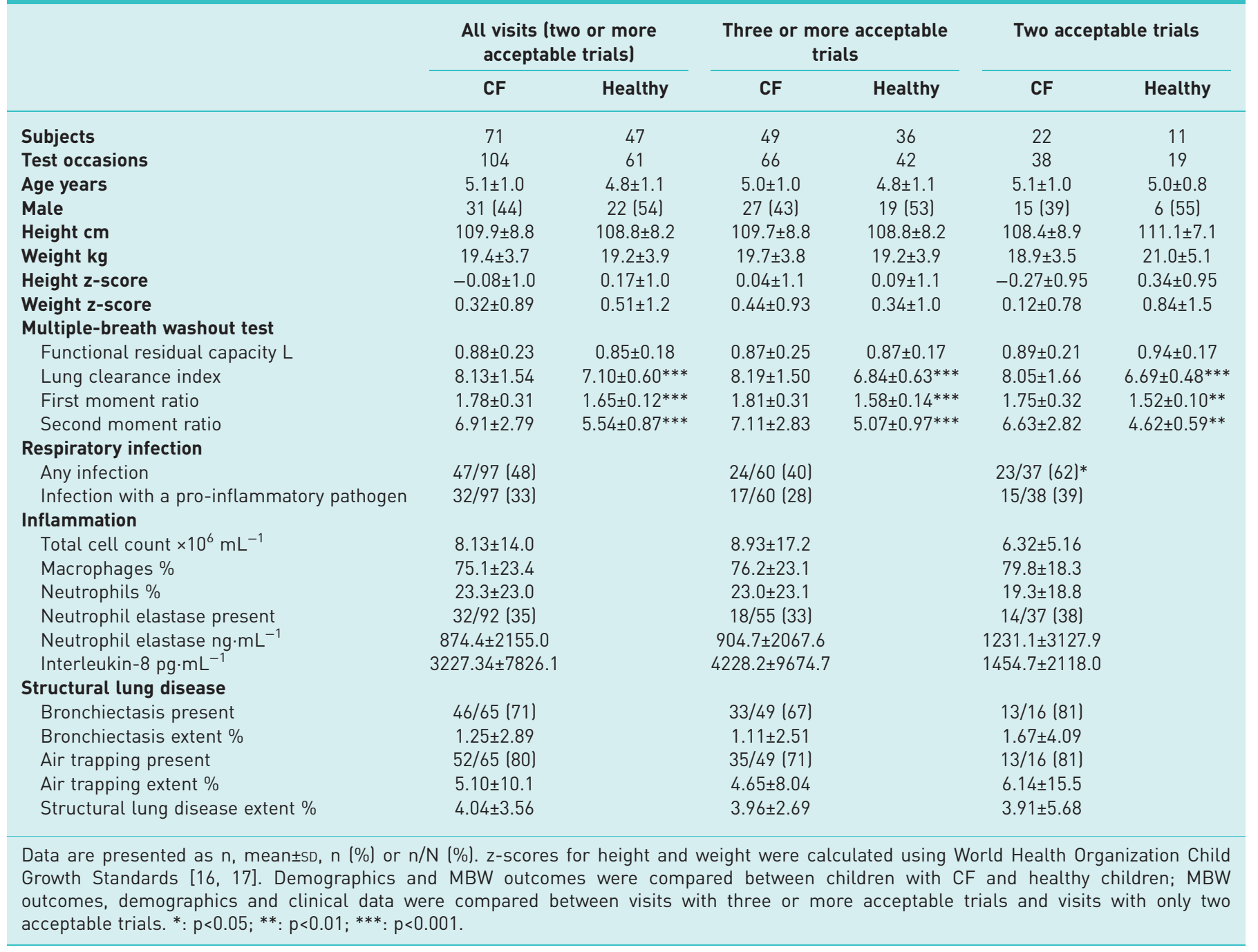

than the feasibility to obtain three acceptable trials $(\mathrm{p}<0.001)$ (figure 1$)$. MBW feasibility was higher in healthy children compared with children with CF $(\mathrm{p}<0.001)$ (table 2).

In the overall population of children with CF who obtained two or more acceptable MBW trials, $48 \%$ had a respiratory infection and $33 \%$ had an infection with a pro-inflammatory pathogen. Neutrophil elastase was detected in $35 \%$ of BAL fluid samples, while bronchiectasis was found to be present in $71 \%$ of chest CT scans (table 1). The presence of a respiratory infection was the only clinical outcome in CF to be significantly different when compared between children who were able to perform three or more MBW trials and children who achieved only two trials (table 1).

\section{Paired analysis between the first two trials and all trials from visits with three or more acceptable MBW trials}

We aimed to determine if MBW outcomes averaged from the first two MBW trials collected from a testing session had the same ability to detect CF lung disease as the MBW outcomes averaged from all the trials in children who obtained three or more MBW trials. We performed a paired statistical analysis comparing MBW outcomes from the first two trials and all trials from an individual testing session in the subset of children who obtained three or more MBW trials.

Mean FRC, LCI and moment ratios calculated from the first two trials were not different to the values calculated from all trials (FRC, $\mathrm{p}=0.89$; LCI, $\mathrm{p}=0.84 ; \mathrm{M} 1 / \mathrm{M} 0, \mathrm{p}=0.95 ; \mathrm{M} 2 / \mathrm{M} 0, \mathrm{p}=0.94$ ) (figure 2 and 
TABLE 2 Success rates of obtaining acceptable and repeatable multiple-breath washout measurements in pre-school-aged children when three or more trials or two or more trials were deemed acceptable

\begin{tabular}{|c|c|c|c|c|}
\hline \multirow{2}{*}{$\begin{array}{l}\text { Age } \\
\text { years }\end{array}$} & \multicolumn{2}{|c|}{ Cystic fibrosis } & \multicolumn{2}{|c|}{ Healthy } \\
\hline & $\begin{array}{l}\text { Three or more } \\
\text { acceptable trials }\end{array}$ & $\begin{array}{c}\text { Two or more } \\
\text { acceptable trials }\end{array}$ & $\begin{array}{l}\text { Three or more } \\
\text { acceptable trials }\end{array}$ & $\begin{array}{c}\text { Two or more } \\
\text { acceptable trials }\end{array}$ \\
\hline 3 & 7/21 (33) & $14 / 21(67)^{*}$ & $10 / 14(71)$ & 13/14 (93) \\
\hline 4 & $18 / 38$ (47) & $27 / 38(71)^{*}$ & 9/17 (53) & 15/17 (88)* \\
\hline 5 & 22/49 (45) & $35 / 49(71) * *$ & $14 / 26(54)$ & $22 / 26$ (85)* \\
\hline 6 & & $28 / 41(68)^{*}$ & $9 / 15(60)$ & 11/15 (73) \\
\hline Overall & $66 / 149(44)$ & $104 / 149(70)^{* * *}$ & 42/72 (58) & $61 / 72(85)^{* * *}$ \\
\hline
\end{tabular}

table 3). There were no differences in the ability of LCI, M1/M0 and M2/M0 calculated from the first two trials to discriminate between children with CF and healthy children compared with values from all trials.

When MBW outcomes were calculated from all MBW trials, $57 \%$ of children with CF had abnormal LCI, $60 \%$ had abnormal M1/M0 and 68\% had abnormal M2/M0 using cut-offs derived from a previous study from our group [3]. When MBW outcomes were calculated from the first two trials, $57 \%$ of children with CF had abnormal LCI, 59\% had abnormal M1/M0 and 65\% had abnormal M2/M0 using the same cut-off values. There were no differences between these proportions when comparing MBW outcomes from all trials versus the first two trials.

There were no significant differences between the coefficients of associations with pulmonary inflammation, infection and structural lung disease between LCI, M1/M0 and M2/M0 calculated from the first two MBW trials and all MBW trials in children who could obtain three or more acceptable trials (table 3).

\section{Group comparison between visits with two or more acceptable trials and three or more acceptable trials}

To determine if changing the MBW acceptability criteria from requiring at least three acceptable trials to two acceptable trials affected clinical utility, we compared MBW outcomes and associations with clinical markers of disease between visits with two or more acceptable trials and visits with three or more acceptable trials. We found no significant differences between MBW outcomes between the two groups.

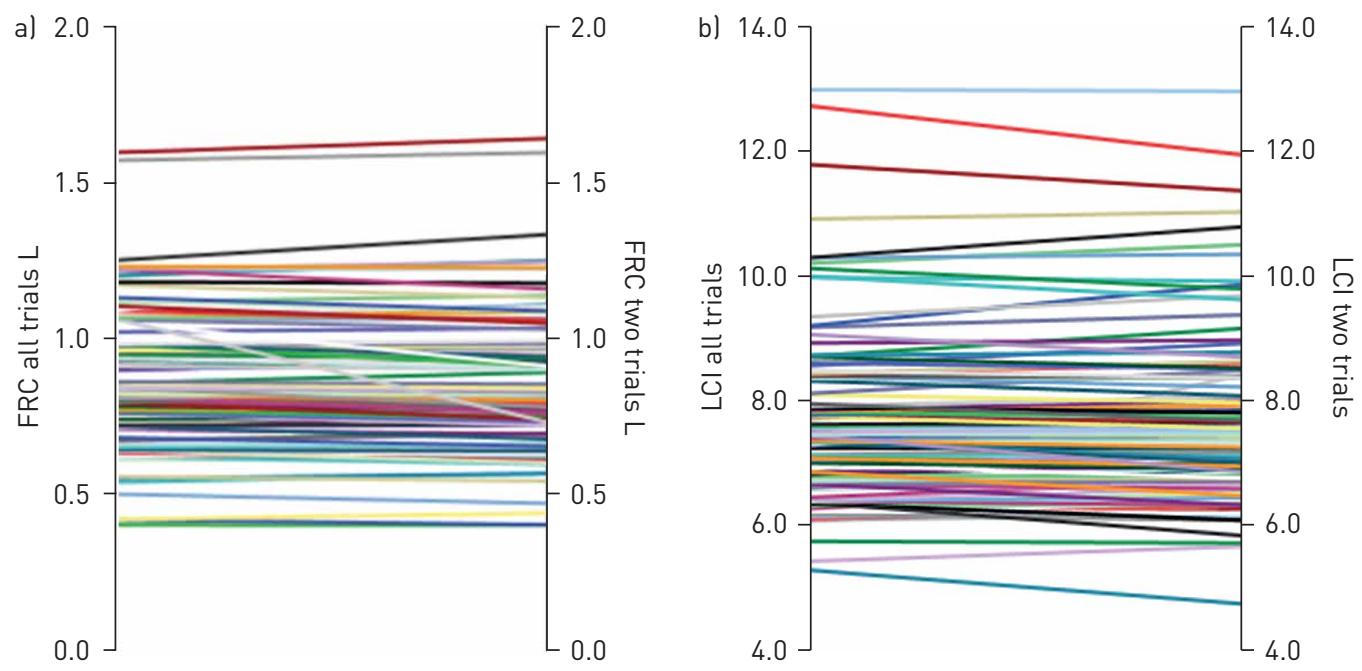

FIGURE 2 Differences in a) functional residual capacity (FRC) and b) lung clearance index (LCI) when using all trials in comparison with only the first two trials from children who completed three or more acceptable trials. A paired t-test was performed to compare the differences in FRC and $\mathrm{LCl}$ values. 
TABLE 3 Relationship between multiple-breath washout (MBW) test outcomes with inflammation and structural lung disease in children with cystic fibrosis when MBW outcomes were calculated from all trials obtained compared with the first two trials obtained in visits with three or more acceptable trials

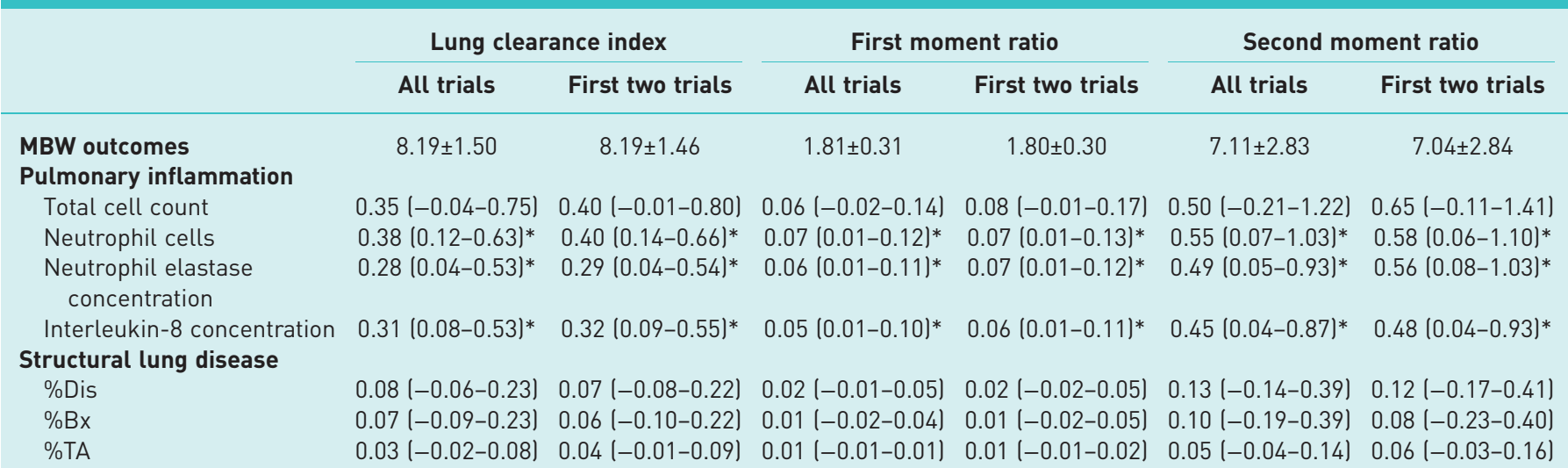

The raw MBW outcomes are presented, and the coefficients for the linear mixed effects model (adjusted for test centre) for associations with markers of pulmonary inflammation and structural lung disease are presented with $95 \%$ confidence intervals. \%Dis: volume proportion of the lung with airway disease; \%Bx: volume proportion of the lung with bronchiectasis; \%TA: volume proportion of the lung with trapped air. *: $p<0.05$.

Children with CF in both groups had significantly higher LCI, M1/M0 and M2/M0 compared with healthy children (table 1).

In both groups there were associations between MBW outcomes with markers of pulmonary inflammation, including neutrophil cells, neutrophil elastase and interleukin-8 concentrations, but not with structural lung disease (table 4). Strengths of associations were stronger in the group with two or more acceptable trials compared with the group with three or more acceptable trials with all markers of pulmonary inflammation. Significant associations were seen in the two or more acceptable trials group for total cell counts, but not in the three or more acceptable trials group. Further testing of the equality of mixed effects model coefficients of association between MBW test outcomes with inflammation and structural lung disease found that the coefficients were not significantly different.

The sensitivity of abnormal MBW outcomes to indicate the presence of a respiratory infection was higher in the group with two or more acceptable trials compared with the group with three or more acceptable trials (table 5). The sensitivity and specificity of abnormal MBW outcomes to indicate the presence of bronchiectasis was higher in the group with two or more acceptable trials compared with the group with three or more acceptable trials.

\section{Discussion}

This study shows that there were no significant differences between MBW outcomes or associations with clinical markers of disease when MBW outcomes are calculated from two acceptable trials versus three or more acceptable trials. MBW outcomes from two trials provide the same ability to discriminate between healthy children and children with $\mathrm{CF}$ as when using MBW outcomes from three trials. In the paired analysis of children with CF, markers of ventilation distribution (LCI, M1/M0 and M2/M0) calculated from the first two trials and all trials had the same ability to detect the presence and extent of pulmonary inflammation, infection and structural lung disease. In addition, changing the acceptability criteria from at least three acceptable trials to two acceptable trials in the entire population significantly improved MBW test feasibility but did not change MBW outcomes or associations with clinical markers of disease in the same cohort. Moreover, the findings from this study suggest that by excluding subjects who are unable to complete three acceptable trials, the ability to detect associations with clinical markers of disease may be diminished due to reduced power. This study demonstrates that results from two acceptable trials provide sufficient information on ventilation distribution if three trials are unable to be obtained.

In this study, we only included children within the pre-school age range, a population known to be particularly challenging for measuring lung function [2]. We show that the success rate of obtaining three or more acceptable and repeatable trials in pre-school age children is $44 \%$ in children with CF and $58 \%$ in healthy children; however, by accepting MBW tests with two acceptable trials this is markedly improved to $70 \%$ in CF and $85 \%$ in controls, therefore significantly improving feasibility of the MBW test in pre-school 
TABLE 4 Group comparisons for the relationship between multiple-breath washout test outcomes with inflammation and structural lung disease in children with cystic fibrosis when results from the first two trials of children who could perform three or more acceptable trials ${ }^{\#}$ were compared with children who could perform two or more acceptable trials"

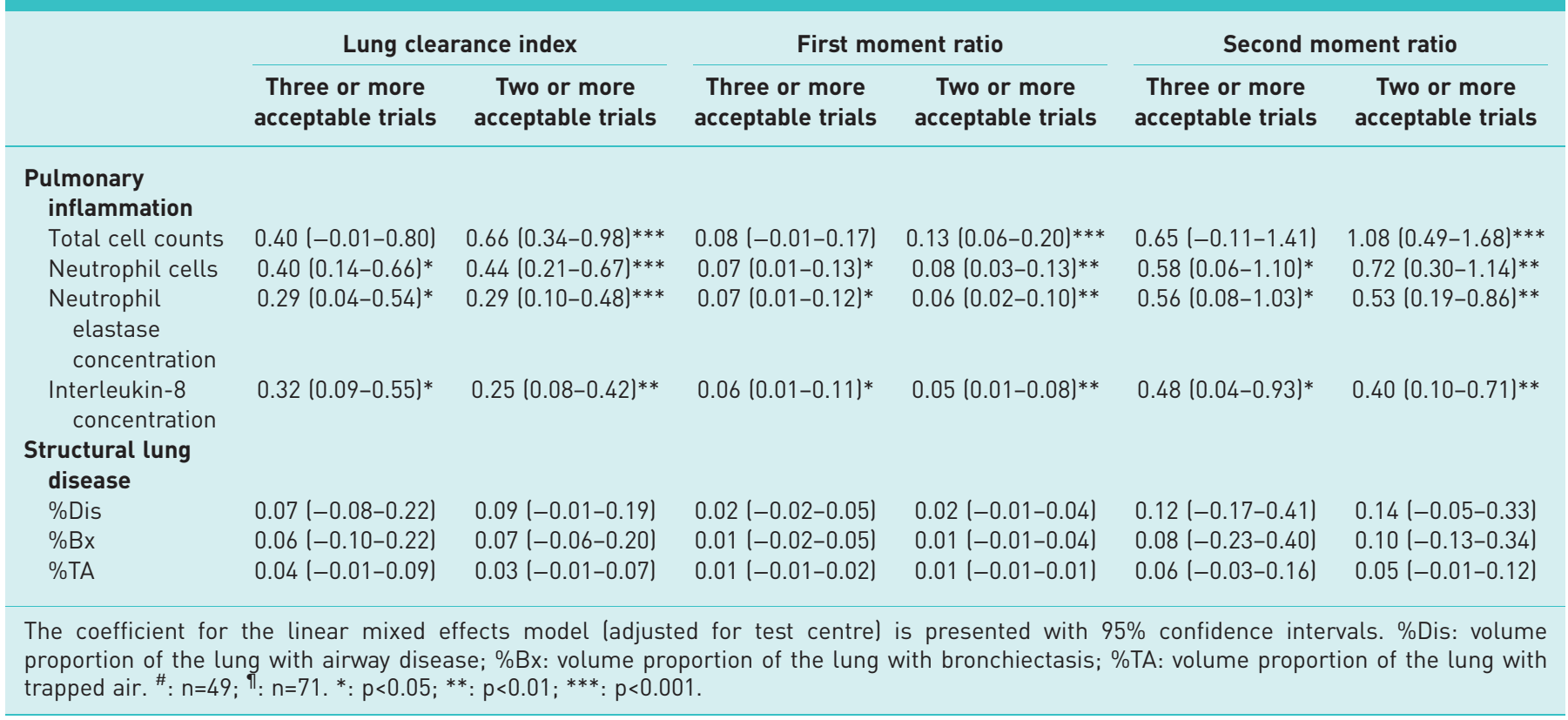

children. In our study, the higher feasibility in healthy children may be attributed to study visits in CF patients being scheduled around other clinical appointments, with limited time $(<30 \mathrm{~min})$ to complete the MBW testing compared with healthy control participants who attended a study visit to solely complete lung function testing. Previous feasibility studies on MBW testing in pre-school children using commercial equipment have also demonstrated higher feasibility when two trials were accepted compared with three trials. Feasibility to obtain three trials was $24 \%$ when the testing time was restricted to $20 \mathrm{~min}$, but this increased to $60 \%$ when two trials were accepted [8]. Another study assessing feasibility of MBW testing in a clinical sitting showed an increase in feasibility with age, with an overall success rate of $80 \%$ in children aged 3-6 years; however, the study only reported acceptability for tests with two or more repeatable trials [9]. Overall, MBW feasibility in our study is comparable to that of previously published studies.

There have been many studies that have aimed to improve MBW test feasibility. Studies suggest that feasibility is higher when using facemasks rather than mouthpieces [18]. An early study of MBW testing in pre-school children showed that the use of a mask to obtain three MBW trials in pre-school children

TABLE 5 Ability of the multiple-breath washout test to detect the presence of neutrophil elastase, respiratory infections and structural lung disease in pre-school children with cystic fibrosis who achieved three or more and two or more acceptable trials

\begin{tabular}{|c|c|c|c|c|c|c|}
\hline \multicolumn{7}{|c|}{ Neutrophil elastase } \\
\hline Sensitivity \% & $83(59-96)$ & $83(59-96)$ & 89 (65-99) & 89 (65-99) & 89 (65-99) & 89 (65-99) \\
\hline Specificity \% & $61(43-76)$ & $64(46-79)$ & $61(43-76)$ & $64(46-79)$ & $47(31-64)$ & $47(30-65)$ \\
\hline Specificity \% & $58(39-75)$ & $56(38-72)$ & $58(39-75)$ & 53 (37-68) & $42(25-61)$ & $42(27-58)$ \\
\hline \multicolumn{7}{|l|}{ Bronchiectasis } \\
\hline Sensitivity \% & $66(46-82)$ & 70 (51-85) & $65(46-82)$ & $69(50-84)$ & 83 (64-94) & 84 (67-95) \\
\hline Specificity \% & 59 (33-82) & $69(41-89)$ & 59 (33-82) & $63(35-85)$ & $53(28-77)$ & 63 (35-85) \\
\hline
\end{tabular}


achieved an overall success rate of 79\% [5]. Feasibility was also higher in healthy children compared with children with CF in this study. A recent publication demonstrated that feasibility could be improved with familiarisation, where at the initial visit, pre-school feasibility was $66 \%$ to achieve two or more trials and this increased to $89 \%$ at subsequent visits [10]. MBW testing was performed using facemasks and the second visit performed 1 month after the initial visit. RoBINson et al. [19] aimed to assess if the MBW test could be shortened by using two trials rather than three trials, and found that although there was a statistically significant difference in mean LCI from three tests and the first two tests in school-aged children, the difference of 0.07 units was not clinically significant. LCI calculated from two technically acceptable tests provided similar abnormal values as from three tests in CF patients. Another study attempted to shorten test duration in school-aged children using three MBW trials with a washout to $1 / 20$ th of the starting concentration or two trials to the standard 1/40th concentration. Similar to findings from RoBINson et al. [19], mean LCI from two trials was similar to LCI from three trials and also had a high sensitivity to detect CF lung disease [20]. A subsequent study, however, showed that while washout to $1 / 20$ th of the starting concentration reduced test duration, it did not improve feasibility in children $<3$ years of age [9]. Reducing washout time may not be suitable in patients with mild disease as there is the potential to miss information on ventilation distribution during later stages of the washout; thus, accepting MBW tests with two technically acceptable trials may be the most feasible means to increase feasibility in young children.

The strengths of this study are that infection and inflammation information from BAL, as well as structural lung disease on chest CT, were determined during the same week as MBW testing in children with CF. Markers of ventilation inhomogeneity therefore reflected the current clinical status of lung health. A limitation of this study was that we did not include data on test duration. Feasibility in children with CF for obtaining three trials was also low, possibly due to time constraints during testing or the use of a mouthpiece rather than a facemask that could potentially reduce feasibility due to leaks, as it is harder to keep a seal on the mouthpiece, reducing study numbers for the comparisons between two and three or more trials. As such, we identified stronger associations between MBW and pulmonary inflammation outcomes in children with CF from the entire cohort, suggesting a stronger effect with increased power.

We recommend that users should collect at least three acceptable MBW trials whenever possible according to the ERS/ATS consensus statement [1]. However, this study provides evidence that MBW data in pre-school children reported from only two acceptable trials provide comparable outcomes, ability to discriminate between health and disease, and associations with clinical markers of CF lung disease as MBW data reported from three acceptable trials. In addition, by excluding MBW tests with less than two trials, valid data points may not be included in study analyses. The acceptability of MBW visits with only two acceptable trials allows for improved feasibility in young children, which may increase participants eligible for longitudinal assessment of MBW outcomes, and clinical trials utilising MBW data as an end-point, thus assisting the transition of MBW into the clinical setting.

\section{Acknowledgements}

We would like to acknowledge Luke Berry (Telethon Kids Institute, Perth, Australia) for performing the differential cell counts and ELISAs for quantification of neutrophil elastase and interleukin-8, as well as the respiratory fellows for performing the BAL and chest CT. We would also like to thank Clara Mok (Telethon Kids Institute, Perth, Australia) for scoring of the chest CT scans using PRAGMA-CF.

Author contributions: All authors made substantial contributions to the conception or design of the work, or the acquisition, analysis or interpretation of data for the work, and drafting the work or revising it critically for important intellectual content. All authors gave final approval of the version to be published and agree to be accountable for all aspects of the work.

\section{References}

1 Robinson PD, Latzin P, Verbanck S, et al. Consensus statement for inert gas washout measurement using multiple- and single-breath tests. Eur Respir J 2013; 41: 507-522.

2 Rosenfeld M, Allen J, Arets BHGM, et al. An official American Thoracic Society workshop report: optimal lung function tests for monitoring cystic fibrosis, bronchopulmonary dysplasia, and recurrent wheezing in children less than 6 years of age. Ann Am Thorac Soc 2013; 10: S1-S11.

3 Ramsey KA, Rosenow T, Turkovic L, et al. Lung clearance index and structural lung disease on computed tomography in early cystic fibrosis. Am J Respir Crit Care Med 2015; 193: 60-67.

4 Owens CM, Aurora P, Stanojevic S, et al. Lung clearance index and HRCT are complementary markers of lung abnormalities in young children with CF. Thorax 2011; 66: 481-488.

5 Aurora P, Bush A, Gustafsson P, et al. Multiple-breath washout as a marker of lung disease in preschool children with cystic fibrosis. Am J Respir Crit Care Med 2005; 171: 249-256.

6 Simpson SJ, Ranganathan S, Park J, et al. Progressive ventilation inhomogeneity in infants with cystic fibrosis after pulmonary infection. Eur Respir J 2015; 46: 1680-1690.

7 Ramsey KA, Foong RE, Grdosic J, et al. Multiple breath washout outcomes are sensitive to inflammation and infection in children with cystic fibrosis. Ann Am Thorac Soc 2017; 14: 1436-1442.

8 Yammine S, Summermatter S, Singer F, et al. Feasibility of nitrogen multiple-breath washout in inexperienced children younger than 7 years. Pediatr Pulmonol 2016; 51: 1183-1190. 
9 Downing B, Irving S, Bingham Y, et al. Feasibility of lung clearance index in a clinical setting in pre-school children. Eur Respir J 2016; 48: 1074-1080.

10 Stanojevic S, Davis SD, Retsch-Bogart G, et al. Progression of lung disease in preschool patients with cystic fibrosis. Am J Respir Crit Care Med 2017; 195: 1216-1225.

11 Jensen R, Stanojevic S, Klingel M, et al. A systematic approach to multiple breath nitrogen washout test quality. PLoS One 2016; 11: e0157523.

12 Sly PD, Brennan S, Gangell C, et al. Lung disease at diagnosis in infants with cystic fibrosis detected by newborn screening. Am J Respir Crit Care Med 2009; 180: 146-152.

13 Gangell C, Gard S, Douglas T, et al. Inflammatory responses to individual microorganisms in the lungs of children with cystic fibrosis. Clin Infect Dis 2011; 53: 425-432.

14 Rosenow T, Oudraad MCJ, Murray CP, et al. PRAGMA-CF. A quantitative structural lung disease computed tomography outcome in young children with cystic fibrosis. Am J Respir Crit Care Med 2015; 191: 1158-1165.

15 Clogg CC, Petkova E, Haritou A. Statistical methods for comparing regression coefficients between models. Am J Sociol 1995; 100: 5 .

16 WHO Multicentre Growth Reference Study Group. WHO Child Growth Standards: Length/Height-for-Age, Weight-for-Age, Weight-for-Length, Weight-for-Height and Body Mass Index-for-Age: Methods and Development. Geneva, WHO, 2006.

17 de Onis M, Onyango A, Borghi E, et al. Development of a WHO growth reference for school-aged children and adolescents. Bull World Health Organ 2007; 85: 661-668.

18 Robinson PD, Hardaker KM, Gustafsson PM, et al. Effect of interface on multiple breath washout feasibility and results in young children. Am J Respir Crit Care Med 2015; 191: A2330.

19 Robinson PD, Stocks J, Aurora P, et al. Abbreviated multi-breath washout for calculation of lung clearance index. Pediatr Pulmonol 2013; 48: 336-343.

20 Yammine S, Singer F, Abbas C, et al. Multiple-breath washout measurements can be significantly shortened in children. Thorax 2013; 68: 586-587. 